\section{INTEGRATING TRADITIONAL CHINESE MEDICINE INTO PRIMARY CARE PRACTICE}

\author{
P.J. Allen
}

\author{
School of Nursing, Yale University, \\ New Haven, CT, USA
}

Background: Complimentary and alternative medicine is used by millions of people to supplement Western medicine practices or in some cultures, substitute for Western medicine. It is imperative that pediatric nurses be knowledgeable concerning health beliefs and practices of the populations they serve. The Asian population continues to grow and immigrants often maintain health beliefs and illness management strategies from their culture.

Purpose: This poster or presentation will describe a unique educational experience between Yale University and Hong Kong Polytechnic University to offer graduate nursing students a comprehensive didactic course and skills laboratory experience in Traditional Chinese Medicine (TCM).

Description: The objectives of the course were to have students compare theories of health and well-being, disease and illness, between Chinese and Western societies, identify how TCM is currently integrated into health care in China and develop beginning skills in traditional Chinese assessment and treatment modalities. Students had opportunities to visit diagnostic skills centers, integrative health clinics, and herbal dispensaries in addition to attending daily lectures.

Findings: Pictures of the educational program and reflections of the eight APRN students who participated in 2010 will be presented. Students were fascinated by the complexity and relevance of the ancient Chinese philosophy of health and illness and were able to identify ways of integrating this knowledge into practice in the United States.

Summary: Traditional Chinese health care practices are commonly used and nursing health care providers must be more sensitive to their use and aware of cultural safety in integration with Western medicine.

\section{EFFECT OF INTRAPARTUM OXYTOCIN ADMINISTRATION ON FIRST LATCH AND BREASTFEEDING BEHAVIOUR}

I. Olza Fernández¹ , A.M. Malalana Martínez², M.Á. Marín Gabriel², F. López Sánchez ${ }^{3}$, M. Durán Duque $^{4}$, B. Martínez Rodríguez ${ }^{4}$, P. Perez Riveiro ${ }^{4}$,

A. Fernández-Cañadas Morillo ${ }^{5}$

${ }^{1}$ Psychiatry, ${ }^{2}$ Pediatrics, ${ }^{3}$ Obstetrics and

Gynecology, ${ }^{4}$ Obstetrics, Hospital Universitario

Puerta de Hierro-Majadahonda, Madrid, ${ }^{5}$ Obstetrics, Hospital Universitario Puerta de Hierro-Majadahonda, Majadahonda, Spain

Background and aims: The effect of the infusion of synthetic oxytocin on newborn behaviour has received little attention although there is a growing body of evidence that suggests it can affect the behaviour of non human mammals both early and in the long term. The impact of exogenous oxytocin on spontaneous breastfeeding has not been thoroughly investigated. The objective of the study is to evaluate the effect of oxytocin used during induced labour on spontaneous first breastfeeding.

Methods: Observational descriptive study approved by Local Ethical Committee. 24 women with their first term pregnancies were studied. All had singleton, healthy pregnancies and epidural analgesia was used. Newborns were vaginal delivered and immediately placed in skin-to-skin contact (SSC) with the mother. Patients were excluded if $5 \mathrm{~min}$ Apgar was $<9$ or no immediately SSC was applied $(n=2)$. Time in minutes between delivery and spontaneous breastfeeding was measured.

Results: Mean GA and birthweight was $39.5 \pm 1.3 w$ and $3326.1 \pm 323.6 \mathrm{~g}$ respectively. Mean oxytocin dose was $2326.9 \pm 1771.9 \mathrm{mUl}$. Median time to achieve first breastfeed was $56.8 \pm 38 \mathrm{~m}$. Six newborns $(27.2 \%)$ get first breastfeed after $1 \mathrm{~h}$ of SSC. No correlation was found between the amount of oxytocin infused during labour and time of first breastfeed $(r=-0.28 ; p=0.19)$. No association was found between amount of oxytocin and delayed first breastfeeding.

Conclusions: Total dose of oxytocin infused during labour may have no influence on spontaneous first breastfeeding. 